\title{
Fatores de risco cardiovascular em idosos de uma comunidade quilombola
}

\author{
Cardiovascular risk factors in older adults of a quilombola community
}

Factores de riesgo cardiovascular en ancianos de una comunidad quilombola

\author{
Paula Gabriella do Nascimento Silva'; Kelliane de Moura Costa"; \\ Ticiane Maria Santos Muniz ${ }^{\prime \prime \prime}$; Ana Larissa Gomes Machado ${ }^{\prime V}$
}

\begin{abstract}
RESUMO
Objetivo: descrever a frequência dos fatores de risco cardiovascular em idosos de uma comunidade quilombola. Método: estudo transversal desenvolvido com idosos quilombolas cadastrados na Estratégia Saúde da Família. A coleta foi realizada com uma amostra de 62 idosos, utilizando-se o teste do qui-quadrado para análise dos dados. Resultados: as frequências dos fatores de risco foram: $67,7 \%$ de hipertensão arterial, 54,8\% de adiposidade abdominal, 22,6\% de glicemia capilar elevada, 19,4\% de excesso de peso, 3,2\% de sedentarismo e 3,2\% de tabagismo, com diferença estatística apenas para adiposidade abdominal em idosas de cor branca $(p<0,05)$. Conclusão: o estudo identificou elevada frequência de fatores de risco cardiovasculares nos idosos quilombolas atendidos na atenção primária à saúde, com destaque para hipertensão arterial, adiposidade abdominal e glicemia capilar elevada. Os resultados apontam a necessidade de melhoria de acesso da comunidade quilombola aos serviços de saúde.
\end{abstract}

Descritores: Idoso; Doenças Cardiovasculares; Fatores de Risco; Atenção Primária à Saúde.

\section{ABSTRACT}

Objective: to describe the frequency of cardiovascular risk factors in the older adults of a quilombola community. Method: in this cross-sectional study of 62 older adults of a quilombo registered with Brazil's Family Health Strategy, the data collected were analyzed using the Chi-square test. Results: risk factor frequencies were: $67.7 \%$ for arterial hypertension; $54.8 \%$, abdominal adiposity; $22.6 \%$, high capillary blood glucose; $19.4 \%$, overweight; $3.2 \%$, sedentary lifestyle; and $3.2 \%$, smoking. Statistical difference was observed only for abdominal adiposity in older, white women $(p<0.05)$. Conclusion: this study identified a high frequency of cardiovascular risk factors in quilombola older adults treated in primary health care, particularly arterial hypertension, abdominal adiposity, and high capillary blood glucose. These results indicate the need to improve quilombola communities' access to health services.

Descriptors: Aged; Cardiovascular Diseases; Risk Factors; Primary Health Care.

\section{RESUMEN}

Objetivo: describir la frecuencia de factores de riesgo cardiovascular en los adultos mayores de una comunidad quilombola. Método: en este estudio transversal de 62 adultos mayores de un quilombo inscrito en la Estrategia de Salud de la Familia de Brasil, los datos recolectados se analizaron mediante la prueba de Chi-cuadrado. Resultados: las frecuencias de los factores de riesgo fueron: 67,7\% para la hipertensión arterial; 54,8\%, adiposidad abdominal; 22,6\%, glucemia capilar elevada; 19,4\%, sobrepeso; $3,2 \%$, sedentarismo; y 3,2\%, tabaquismo. Se observó diferencia estadística solo para la adiposidad abdominal en mujeres blancas mayores $(p<0.05)$. Conclusión: este estudio identificó una alta frecuencia de factores de riesgo cardiovascular en adultos mayores quilombolas tratados en atención primaria de salud, particularmente hipertensión arterial, adiposidad abdominal y glucemia capilar alta. Estos resultados indican la necesidad de mejorar el acceso de las comunidades quilombolas a los servicios de salud.

Descriptores: Anciano; Enfermedades Cardiovasculares; Factores de Riesgo; Atención Primaria de Salud.

\section{INTRODUÇÃO}

O envelhecimento, considerado um processo fisiológico, progressivo e dinâmico caracteriza-se por modificações orgânicas que podem resultar em redução da capacidade de adaptação do organismo, ocasionando uma série de complicações à saúde.

É notório que o avanço da idade leva à convivência com doenças crônicas, incapacitantes, que podem afetar a autonomia das pessoas. Entre os idosos brasileiros, em 2013, apenas 22,3\% declararam não apresentar nenhuma doença crônica. Aproximadamente a metade, $48,6 \%$, declarou ter uma ou duas doenças e $29,1 \%$, três ou mais. Dentre as doenças relatadas, predominaram as doenças cardiovasculares ${ }^{1}$.

'Enfermeira. Especialista em Saúde Coletiva. Universidade Federal do Piauí. Picos, Brasil. E-mail: npaulagabriella@yahoo.com. ORCID: https://orcid.org/0000-0003-2859-1917 "Enfermeira. Especialista em Saúde Pública. Secretaria Municipal de Saúde. Picos, Piauí, Brasil. E-mail: kellianecm@hotmail.com. ORCID: https://orcid.org/0000-0002-3245-3771 I"Enfermeira. Especialista em Urgência e Emergência. Universidade Federal do Piauí. Picos, Brasil. E-mail: tmuniz the@hotmail.com. ORCID: https://orcid.org/0000-0001-8810-2797 IVEnfermeira. Doutora. Universidade Federal do Piauí. Picos, Brasil. E-mail: analarissa2001@yahoo.com.br. ORCID: https://orcid.org/0000-0002-7937-6996 
A associação entre a longevidade e o aumento de doenças crônicas é conhecida na literatura apontando para uma maior morbidade por doenças cardiovasculares (DCV) em indivíduos mais velhos. As DCV estão crescendo rapidamente devido aos hábitos de vida inadequados, aumentando-se assim a necessidade de conhecer a situação de saúde e os fatores de risco envolvidos no surgimento destas doenças ${ }^{2}$.

As DCV são exemplos de doenças complexas, isto é, aquelas que apresentam um componente genético e ambiental na sua etiologia. Estudo documenta que mesmo depois de controlar os principais fatores sociodemográficos, a etnia foi significativamente associada à maioria dos resultados adversos nos indicadores de saúde ${ }^{3}$. As doenças cardiovasculares são hoje as maiores causas de morte nas cinco regiões brasileiras e são mais prevalentes entre as pessoas de baixa renda ${ }^{4}$.

Segundo estudo que relacionou os fatores de risco cardiovascular observou-se que os pardos e negros são os grupos mais vulneráveis, estes numerosos nas comunidades quilombolas ${ }^{5}$.

As comunidades quilombolas envolvem a participação de grupos étnico-raciais segundo o critério de autoatribuição, com relações territoriais específicas e com presunção de ancestralidade negra relacionada com a resistência à opressão histórica ${ }^{6}$. Os quilombos foram formados durante o período de escravidão no Brasil, embora atualmente os quilombos não se refiram necessariamente apenas a este tipo de ocupação 7 .

A trajetória histórica da população negra no Brasil mostra que esses indivíduos vêm lutando por melhores condições de saúde e qualidade de vida (QV). Diante das diversas manifestações e frente às desigualdades raciais do Brasil, criou-se a Política Nacional de Saúde Integral da População Negra (PNSIPN) que, dentre seus objetivos prevê a ampliação do acesso da população negra, em particular das comunidades quilombolas, às ações e aos serviços de saúde ${ }^{8}$.

Geralmente, as comunidades quilombolas localizam-se em meio rural, possuem altos índices de analfabetismo e apresentam precárias condições de vida, saneamento, moradia e acesso aos serviços de saúde. Ademais, esses grupamentos étnicos passaram por um processo histórico de expropriação de cultura e de direitos, refletindo nos seus indicadores de saúde ${ }^{7}$. Dada à persistência de disparidades raciais, evidências sistemáticas indicam que os negros têm maior incidência de doenças e morrem mais precocemente, em todas as idades 3 .

Nota-se uma intensificação de ações sociais e políticas destinadas a essas comunidades, porém, apesar do aumento dessas ações as pesquisas ainda demonstram que os indicadores de desenvolvimento humano nestes locais são desiguais e caracterizados como inferior, em relação à sociedade em geral ${ }^{6}$.

Frente ao exposto, objetivou-se descrever a frequência dos fatores de risco cardiovascular em idosos de uma comunidade quilombola.

\section{MÉTODO}

Estudo descritivo com delineamento transversal, realizado em uma comunidade quilombola, localizada na zona rural de um município do interior do Piauí ${ }^{8}$. A comunidade dispõe dos serviços ofertados pela Estratégia de Saúde da Família (ESF), dentre eles: a consulta médica, consulta de enfermagem, acompanhamento ao hipertenso e diabético, visita do agente comunitário de saúde e visitas domiciliárias. Desse modo, a equipe da ESF proporcionou suporte para a coleta de dados, sendo visitados todos os domicílios da área adscrita que possuíam idosos.

A população do estudo foi composta por 62 idosos, sendo adotados os seguintes critérios de inclusão: ter 60 anos ou mais de idade, residir na comunidade quilombola, não estar acamado e ser cadastrado na Unidade de Saúde da Família (USF). Os 62 idosos residentes na comunidade atenderam aos critérios de inclusão sendo, portanto, todos ncluídos na amostra.

Antes da coleta de dados, realizou-se um levantamento dos idosos residentes na comunidade através do cadastro existente na USF na qual os participantes são atendidos e acompanhados. Essa etapa ocorreu no período de agosto a outubro de 2018 por meio de visita domiciliar com aferição da pressão arterial, glicemia capilar (ao acaso) e obtenção das medidas antropométricas como: peso, altura, circunferência abdominal (CA) e cálculo do índice de massa corporal (IMC).

Para a coleta de dados foi utilizado um instrumento composto pelas variáveis sociodemográficas (idade, sexo, situação conjugal, raça, renda, escolaridade e ocupação) e os fatores de risco cardiovascular (tabagismo, etilismo, sedentarismo, obesidade, glicemia capilar e hipertensão arterial).

Em relação ao tabagismo, questionou-se os participantes da amostra quanto ao hábito de fumar e foi considerado fumante o idoso que referiu ser tabagista no momento da entrevista. Para o etilismo (de forma similar), os participantes foram indagados se faziam uso de bebidas alcoólicas até aquele momento. Quanto ao sedentarismo, foi identificado naqueles que responderam não praticar atividade física. Para os idosos que afirmaram praticar, perguntou-se a frequência e o tipo de atividade praticada: se caminhada, ciclismo natação, hidroginástica, dança ou o simples pedalar da bicicleta ${ }^{9}$. 
A obesidade foi classificada mediante valores do peso e altura e posteriormente foi aplicado o cálculo de Índice de Massa Corporal (IMC). Utilizou-se a fórmula IMC=Peso(Kg)/[Altura $]^{2}(\mathrm{~m})$, foi classificado como obeso o idoso que apresentou o IMC $\geq 30 \mathrm{Kg} / \mathrm{m} 2 .{ }^{7}$ Para mensuração do peso foi utilizada balança eletrônica Even ${ }^{\circledR}$ com capacidade total de $300 \mathrm{~kg}$, o participante foi posicionado descalço, com o mínimo de roupas e adereços. A altura foi aferida com o uso do estadiômetro portátil, os voluntários foram colocados em posição ortostática, com a cabeça erguida e os braços estendidos ao longo do corpo.

Para medição da CA utilizou-se fita métrica inextensível de 1,50 metros, graduada de 0,5 em 0,5 centímetros. Essa foi posicionada no ponto médio entre o rebordo costal e a crista ilíaca. Considerou-se CA aumentada quando o valor apresentado foi $\geq 94 \mathrm{~cm}$ para homens e $\geq 80 \mathrm{~cm}$ para mulheres ${ }^{10}$.

A pressão arterial (PA) dos voluntários foi verificada por meio do uso de estetoscópio e esfigmomanômetro, por método indireto, com manguitos de tamanhos apropriados. Foram realizadas três medidas de PA (com intervalo de um minuto entre cada aferição). As mensurações foram realizadas ao final da entrevista, visando garantir que os indivíduos estivessem em repouso, sentados, com as pernas descruzadas, os pés apoiados no chão, o dorso recostado na cadeira relaxado, com o braço esquerdo apoiado sobre a mesa à altura do coração, com a palma da mão voltada para cima, sem que as roupas estivessem garroteando os membros. Além do mais, certificou-se que os mesmos não estivessem com a bexiga cheia, praticado exercícios físicos há pelo menos 60 minutos, ingerido bebidas alcoólicas, café ou alimento e fumado nos 30 minutos anteriores. A PA elevada foi definida como PA sistólica $\geq 140 \mathrm{mmHg}$ e PA diastólica $\geq 90 \mathrm{mmHg}^{11}$.

A glicemia capilar foi realizada ao acaso utilizando o glicosímetro da marca On Call $^{\oplus}$ Plus, além de uma lanceta estéril para coletar uma gota de sangue do dedo dos participantes. Foram considerados idosos com glicemia capilar alterada aqueles que apresentaram um resultado $\geq 200 \mathrm{mg} \backslash \mathrm{dl}^{12}$.

Os dados foram tabulados e analisados pelo IBM SPSS Statistics ${ }^{\circledR}$ versão 20.0 a partir da distribuição de frequência e empregado o teste de qui-quadrado para verificar as diferenças estatísticas. Adotou-se o $p<0,05$ como valor de referência para a significância estatística.

Garantindo os preceitos éticos, os participantes da pesquisa foram abordados em seus domicílios, sendo-Ihes apresentados os objetivos da pesquisa e o Termo de Consentimento Livre e Esclarecido. Portanto, somente após o consentimento dos idosos e assinatura do referido termo, a coleta de dados teve início. O projeto foi aprovado pelo Comitê de Ética em Pesquisa, com o parecer de no. 2.746.707.

\section{RESULTADOS}

De acordo com a caracterização sociodemográfica dos idosos, observou-se que a maioria era do sexo feminino (56,5\%), na faixa etária de $70-79$ anos $(50 \%)$, casada $(67,7 \%)$, sem ocupação $(64,5 \%)$, católica $(93,5 \%)$, com cor da pele negra $(51,6 \%)$, analfabeta $(66,1 \%)$ e com renda de um salário mínimo (100\%).

Os fatores de risco cardiovasculares mais prevalentes entre os idosos foram hipertensão arterial (67,7\%), CA aumentada (54,8\%) e glicemia capilar alterada (22,6\%), como se observa na Tabela 1.

TABELA 1: Distribuição dos fatores de risco cardiovascular, segundo o sexo dos idosos ( $\mathrm{n}=62)$. PicosPI, Brasil, 2018

\begin{tabular}{|c|c|c|c|c|c|c|c|c|}
\hline \multirow{3}{*}{ Fatores de risco } & & \multicolumn{4}{|c|}{ Sexo } & \multirow{3}{*}{ p* } & \multirow{2}{*}{\multicolumn{2}{|c|}{ Total }} \\
\hline & & \multicolumn{2}{|c|}{ Masculino } & \multicolumn{2}{|c|}{ Feminino } & & & \\
\hline & & $\mathbf{n}$ & $\%$ & $\mathbf{n}$ & $\%$ & & $\mathbf{n}$ & $\%$ \\
\hline \multirow[t]{2}{*}{ Tabagismo } & Sim & 1 & 1,6 & 1 & 1,6 & $0,685^{* *}$ & 2 & 3,2 \\
\hline & Não & 26 & 41,9 & 34 & 54,8 & & 60 & 96,8 \\
\hline \multirow[t]{2}{*}{ Sedentarismo } & Sim & - & - & 2 & 3,2 & $0,500 * *$ & 2 & 3,2 \\
\hline & Não & 27 & 43,5 & 33 & 53,2 & & 60 & 96,8 \\
\hline \multirow[t]{2}{*}{ Obesidade } & Sim & 5 & 8,1 & 7 & 11,3 & $0,884^{*}$ & 12 & 19,4 \\
\hline & Não & 22 & 35,5 & 28 & 45,2 & & 50 & 80,6 \\
\hline \multirow[t]{2}{*}{ CA aumentada } & Sim & 8 & 12,9 & 26 & 41,9 & $<0,001^{*}$ & 34 & 54,8 \\
\hline & Não & 19 & 30,6 & 9 & 14,5 & & 28 & 45,2 \\
\hline \multirow[t]{2}{*}{ PA elevada } & Sim & 15 & 24,2 & 27 & 43,5 & $0,071^{*}$ & 42 & 67,7 \\
\hline & Não & 12 & 19,4 & 8 & 12,9 & & 20 & 32,3 \\
\hline \multirow[t]{2}{*}{ Glicemia capilar alterada } & Sim & 6 & 9,7 & 8 & 12,9 & $0,953^{*}$ & 14 & 22,6 \\
\hline & Não & 21 & 33,9 & 27 & 43,5 & & 48 & 77,4 \\
\hline
\end{tabular}

${ }^{*} x^{2}$ de Pearson; ${ }^{* *}$ Teste Exato de Fisher. 
As frequências de hipertensão arterial, adiposidade abdominal, glicemia capilar, excesso de peso, sedentarismo e tabagismo foram, respectivamente, de $43,5 \%$ para idosas e $24,2 \%$ para idosos ( $p>0,05$ ), $41,9 \%$ para idosas e $12,9 \%$ para idosos ( $p<0,05), 12,9 \%$ para idosas e $9,7 \%$ para idosos ( $p>0,05), 11,3 \%$ em idosas e $8,1 \%$ em idosos $(p>0,05), 3,2 \%$ em idosas $(p>0,05), 1,6 \%$ em idosas e $1,6 \%$ em idosos ( $p>0,05)$.

A Tabela 2 mostra que os FRCV foram mais frequentes em idosos na faixa etária de 70 -79 anos, quando comparados com as outras faixas, entre eles o sedentarismo (50,0\%), a hipertensão (38,7\%) e a CA aumentada (32,3\%). Não foi observada associação estatística significativa entre a faixa etária dos idosos e os FRCV.

TABELA 2: Distribuição dos fatores de risco cardiovascular, segundo a faixa etária $(\mathrm{n}=62)$. Picos-PI, Brasil, 2018

\begin{tabular}{|c|c|c|c|c|c|c|c|c|c|c|}
\hline \multirow{3}{*}{ Fatores de risco } & & \multicolumn{6}{|c|}{ Faixa etária } & \multirow{3}{*}{ p* } & \multirow{2}{*}{\multicolumn{2}{|c|}{ Total }} \\
\hline & & \multicolumn{2}{|c|}{60 -69 } & \multicolumn{2}{|c|}{$70-79$} & \multicolumn{2}{|c|}{80 e mais } & & & \\
\hline & & $\mathrm{n}$ & $\%$ & $\mathrm{n}$ & $\%$ & $\mathbf{n}$ & $\%$ & & $\mathrm{n}$ & $\%$ \\
\hline \multirow[t]{2}{*}{ Tabagismo } & Sim & - & - & 2 & 3,2 & - & - & 0,356 & 2 & 3,2 \\
\hline & Não & 24 & 38,7 & 29 & 46,8 & 7 & 11,3 & & 60 & 96,8 \\
\hline \multirow[t]{2}{*}{ Sedentarismo } & Sim & 22 & 35,5 & 31 & 50,0 & 7 & 11,3 & 0,195 & 60 & 96,8 \\
\hline & Não & 2 & 3,2 & - & - & - & - & & 2 & 3,2 \\
\hline \multirow[t]{2}{*}{ Obesidade } & Sim & 6 & 9,7 & 4 & 6,5 & 2 & 3,2 & 0,428 & 12 & 19,4 \\
\hline & Não & 18 & 29,0 & 27 & 43,5 & 5 & 8,1 & & 50 & 80,6 \\
\hline \multirow[t]{2}{*}{ CA aumentada } & Sim & 11 & 17,7 & 20 & 32,3 & 3 & 4,8 & 0,307 & 34 & 54,8 \\
\hline & Não & 13 & 21 & 11 & 17,7 & 4 & 6,5 & & 28 & 45,2 \\
\hline \multirow[t]{2}{*}{ PA elevada } & Sim & 14 & 22,6 & 24 & 38,7 & 4 & 6,5 & 0,264 & 42 & 67,7 \\
\hline & Não & 10 & 16,1 & 7 & 11,3 & 3 & 4,8 & & 20 & 32,3 \\
\hline \multirow[t]{2}{*}{ Glicemia capilar alterada } & Sim & 4 & 6,5 & 7 & 11,3 & 3 & 4,8 & 0,345 & 14 & 22,6 \\
\hline & Não & 20 & 32,3 & 24 & 38,7 & 4 & 6,5 & & 48 & 77,4 \\
\hline
\end{tabular}

${ }^{*} x^{2}$ de Pearson

Em relação à associação da cor da pele com os FR, observou-se que houve associação estatística significativa apenas com a CA aumentada $(p=0,003)$. Dentre os fatores de risco investigados, o sedentarismo $(51,6 \%)$ e a PA elevada $(40,3 \%)$ foram os mais prevalentes entre os idosos com a cor da pele negra (Tabela 3 ).

TABELA 3: Associação dos fatores de risco cardiovascular com a cor da pele dos idosos. Picos-PI, 2018

\begin{tabular}{|c|c|c|c|c|c|c|c|c|c|c|}
\hline \multirow{3}{*}{ Fatores de risco } & & \multicolumn{6}{|c|}{ Cor da pele } & \multirow{3}{*}{$\mathbf{p}^{*}$} & \multirow{2}{*}{\multicolumn{2}{|c|}{ Total }} \\
\hline & & \multicolumn{2}{|c|}{ Negro } & \multicolumn{2}{|c|}{ Branco } & \multicolumn{2}{|c|}{ Outro } & & & \\
\hline & & $\mathbf{n}$ & $\%$ & $\mathrm{n}$ & $\%$ & $\mathbf{n}$ & $\%$ & & $\mathbf{n}$ & $\%$ \\
\hline \multirow[t]{2}{*}{ Tabagismo } & Sim & 1 & 1,6 & - & - & 1 & 1,6 & $* 0,186$ & 2 & 3,2 \\
\hline & Não & 31 & 50,0 & 24 & 38,7 & 5 & 1,6 & & 60 & 96,8 \\
\hline \multirow[t]{2}{*}{ Sedentarismo } & Sim & 32 & 51,6 & 23 & 37,1 & 5 & 8,1 & *0,139 & 60 & 96,8 \\
\hline & Não & 0 & & 1 & 1,6 & 1 & 1,6 & & 2 & 3,2 \\
\hline \multirow[t]{2}{*}{ Obesidade } & Sim & 3 & 4,8 & 7 & 11,3 & 2 & 3,2 & $* 0,111$ & 12 & 19,4 \\
\hline & Não & 29 & 46,8 & 17 & 27,4 & 4 & 6,5 & & 50 & 80,6 \\
\hline \multirow[t]{2}{*}{ CA aumentada } & Sim & 11 & 17,7 & 18 & 29,0 & 5 & 8,1 & $* * 0,003$ & 34 & 54,8 \\
\hline & Não & 21 & 33,9 & 6 & 9,7 & 1 & 1,6 & & 28 & 45,2 \\
\hline \multirow[t]{2}{*}{ PA elevada } & Sim & 25 & 40,3 & 14 & 22,6 & 3 & 4,8 & $* 0,180$ & 42 & 67,7 \\
\hline & Não & 7 & 11,3 & 10 & 16,1 & 3 & 4,8 & & 20 & 32,3 \\
\hline \multirow[t]{2}{*}{ Glicemia capilar alterada } & Sim & 6 & 9,7 & 7 & 11,3 & 1 & 1,6 & $* 0,616$ & 14 & 22,6 \\
\hline & Não & 26 & 81,2 & 17 & 70,8 & 5 & 83,3 & & 48 & 77,4 \\
\hline
\end{tabular}

* Teste razão de verossimilhança; **Teste qui-quadrado

\section{DISCUSSÃO}

O maior percentual de idosos do sexo feminino identificado na pesquisa corrobora com dados publicados em outros estudos $^{13,14}$. Segundo o Censo Demográfico de 2010, pessoas do sexo feminino vivem em média 7,6 anos a mais que os homens ${ }^{8}$. 
O predomínio de mulheres em praticamente todas as faixas etárias evidenciado com o aumento da expectativa de vida desse gênero, vem se acentuando no Brasil em diversos estudos de bases populacionais, como a Pesquisa Nacional por Amostras de Domicílios $(P N A D)^{15}$. Os resultados da pesquisa replicam-se mundialmente, provavelmente por mortalidade precoce entre os homens, resultando assim em maior expectativa de vida entre as mulheres, isso pode justificar o maior número de mulheres nos estudos realizados com idosos.

O predomínio de uma população idosa na faixa etária de 70 a 79 anos foi correspondente ao inquérito realizado com idosos de 15 capitais brasileiras e o Distrito Federal $(55,4 \%)^{16}$. Esse fato pode influenciar nas condições de saúde, visto que pessoas muito idosas são em sua maioria mais frágeis e precisam de serviços de maior complexidade.

Em relação ao estado civil a maioria dos idosos investigados era casada, resultado semelhante ao encontrado no estudo que investigou os FR em idosos de Minas Gerais ${ }^{17}$. O fato dos idosos serem casados pode aumentar a chance de adesão ao tratamento das doenças cardiovasculares, pois se sabe que o apoio familiar é de extrema importância no tratamento de doenças e na prevenção de agravos ${ }^{18}$.

Acerca da escolaridade, 66,1\% dos participantes declararam nunca ter estudado, resultado superior ao encontrado no estudo sobre FR cardiovascular em idosos no interior de Minas Gerais ${ }^{17}$. A baixa escolaridade dos idosos quilombolas pode ser justificada pela história da escravidão do povo negro, que por muitos anos ficaram à margem da sociedade, tendo muitos dos seus direitos negados e por se tratar de uma comunidade rural, a baixa escolaridade pode estar associada à dificuldade de acesso às escolas.

A baixa renda entre os idosos pode ser um fator que dificulta na prevenção e controle dos FR cardiovasculares, assim como na adoção de hábitos de vida saudáveis.

Na variável cor da pele $51,6 \%$ dos entrevistados se autodeclararam de cor negra, esse resultado condiz com estudo realizado com idosos na Bahia ${ }^{19}$ que apresentou $56 \%$ de pessoas da cor negra. Algumas transformações têm marcado a composição racial da população idosa brasileira, pois havia no ano 2000 um maior número de idosos brancos, seguidos pelos pardos e pretos, e em 2010 , segundo o censo demográfico brasileiro ${ }^{8}$, diminuiu a proporção de idosos brancos e aumentou o número de pardos e pretos.

A iniquidade quanto ao acesso ao sistema de saúde percebida na comunidade negra é consequência da negligência secular aos direitos sociais, o que espelha na redução do bem-estar desse público durante o processo de envelhecimento ${ }^{13}$.

Em relação ao estilo de vida, a alta frequência de sedentarismo entre os idosos corrobora com os achados de uma investigação realizada em Floriano-PI que identificou $74,8 \%$ dos idosos sedentários ${ }^{17}$. Outro estudo realizado em comunidades quilombolas localizadas no norte de Minas Gerais, também apresentou associação positiva $(p<0,001)$ entre o sedentarismo e desenvolvimento de DCNT, predominantemente no sexo masculino. 0 sedentarismo identificado em idosos pertencentes às comunidades quilombolas pode estar associado a fatores como desigualdade social, qualidade de vida limitada, baixa escolaridade, acesso carente à informação, fatores que refletem a vulnerabilidade em inúmeros âmbitos deste grupo social ${ }^{20}$

Em relação à obesidade central houve um percentual de $54,8 \%$ entre os idosos investigados, sendo o FR mais prevalente no sexo feminino $(45,2 \%)$ e nos idosos de cor negra $(51,6 \%)$, resultado que converge com uma pesquisa realizada em Vitória da Conquista -BA com quilombolas na qual se evidenciou a prevalência de obesidade com um percentual superior entre as mulheres ${ }^{21}$.

A obesidade central representa uma relevante questão no cenário da saúde que pode estar associada, mesmo que em parte, à transição nutricional bem como às transformações demográficas e epidemiológicas. Além do mais, sua predominância entre as mulheres provavelmente deve-se ao acúmulo de gordura natural na região abdominal provocado pelas disfunções metabólicas e hormonais frequentes no sexo feminino no decorrer dos anos ${ }^{22}$.

O acúmulo de gordura abdominal em pessoas do sexo feminino está relacionado com a mudança no metabolismo que acompanha o climatério e ocorre à custa da redução da lipase lipoprotéica, responsável, juntamente com o estrogênio, por regular o acúmulo de gordura e sua distribuição nos tecidos. Há uma tendência ao depósito de gordura perivisceral, com o desenvolvimento de um padrão androide de distribuição de gordura ${ }^{23}$

O FR hipertensão arterial apresentou alta frequência entre os idosos, apresentando um percentual de $67,7 \%$, resultado semelhante ao encontrado em outra pesquisa ${ }^{4}$ e superior aos achados de um estudo realizado em Porto Alegre-RS, com um percentual de $40 \%$ de idosos com hipertensão arterial ${ }^{24}$. A HA é um agravo de alta prevalência nacional que muitas vezes, apresenta comorbidades associadas e um elevado risco de mortalidade. É considerada um dos maiores fatores de risco para as doenças cardiovasculares, com repercussões na qualidade de vida dos indivíduos ${ }^{4}$.

A prevalência de idosos do sexo feminino (43,5\%) com pressão arterial aumentada foi maior que os idosos do sexo masculino (24,2\%). Esse dado diverge de um estudo realizado no Rio Grande do Sul em que a hipertensão foi 
superior entre os homens, sendo a HAS o principal FR para o surgimento de doenças cardiovasculares ocasionado pela soma de outros fatores preditores de risco de evento cardiovascular como obesidade e dislipidemias ${ }^{24}$.

Um estudo transversal, realizado no estado do Sergipe demonstra que existe uma prevalência elevada da HAS (26\%) nas comunidades quilombolas ao serem comparados às estimativas da população geral (20,4\%) do referido estado. Somado a esse dado, pesquisas em população geral desenvolvidas no Brasil, assim como em outros países apontam a prevalência de HAS conforme o sujeito envelhece. Outra evidência relevante é que a comunidade de cor negra demonstra maior predisposição à rigidez arterial em relação às demais etnias ${ }^{14}$

É importante destacar que entre os idosos com pressão arterial elevada, 59,5\% eram de cor negra. Nos negros, a prevalência e a gravidade da hipertensão são maiores, o que pode estar relacionado a fator étnicos elou socioeconômicos. No Brasil predominam os miscigenados, que podem diferir dos negros quanto às características da hipertensão ${ }^{16}$

A limitação do estudo consistiu no difícil acesso às residências dos idosos quilombolas, fato que está intrinsecamente relacionado à baixa procura dessa comunidade pelos atendimentos ofertados na USF. Ratificando assim, a necessidade dos quilombolas serem enxergados a partir de um olhar equitativo, pois é um público historicamente cercado de iniquidades (seja pela precariedade presente na zona rural com a falta de saneamento básico, a dificuldade de acesso aos serviços de saúde, a baixa renda) ou até mesmo pelos resquícios de uma herança cultural que marginalizou social, cultural e economicamente essa comunidade.

\section{CONCLUSÃO}

O estudo identificou elevada frequência de fatores de risco cardiovasculares nos idosos quilombolas atendidos na atenção primária à saúde, com destaque para hipertensão arterial, adiposidade abdominal e glicemia capilar. Os resultados apontam a necessidade de melhoria de acesso da comunidade quilombola aos serviços de saúde.

$\mathrm{O}$ atendimento humanizado e singular dos quilombolas assegurando o acesso às ações e aos serviços de saúde de forma eficaz deve fazer parte das ações realizadas pelas equipes da atenção primária à saúde, as quais realizam o acompanhamento cardiovascular contínuo dos idosos residentes na zona rural.

\section{REFERÊNCIAS}

1. Bacal F, Zimerman LI, Caramori PRA, Lemos PA, Hajjar LA, Feitosa-Filho G, Peixoto JM, Pinheiro JES. Atualização das Diretrizes em Cardiogeriatria da Sociedade Brasileira de Cardiologia - 2019. Arq. Bras. Cardiol. [Internet], 2019 [cited 2020 Jul 25 ]; 112 (5): 649-705. Available from: http://publicacoes.cardiol.br/portal/abc/portugues/2019/v11205/pdf/11205024.pdf.

2. Massa KHC, Duarte YAO, Chiavegatto Filho ADP. Analysis of the prevalence of cardiovascular diseases and associated factors among the elderly, 2000-2010. Ciênc. saúde coletiva [Internet], 2019 [cited 2020 Jul 25]; 24 (1): 105-14. DOI: https://doi.org/10.1590/1413-81232018241.02072017.

3. Judy HNG, Bierman AS, Elliot MN, Wilson RG, Xia C, Scholle SH. Beyond black and white: race/ethnicity and health status among older adults. Am. J. Manag. Care [Internet], 2014 [cited 2020 Jul 25]; 20 (3): 239-48. Available from: https://www.ncbi.nlm.nih.gov/pmc/articles/PMC4474472/.

4. Malta DC, Andrade SSCA, Oliveira TP, Moura L, Prado RR, Souza MFM. Probability of premature death for chronic noncommunicable diseases, Brazil and Regions, projections to 2025. Rev. Bras. Epidemiol. [Internet], 2019 [cited 2019 Jun 12]; 22: E190030. DOI: https://doi.org/10.1590/1980-549720190030.

5. Toledo NN, Almeida GS, Matos MMM, Balieiro AAS, Martin LC, Franco RJS, Mainbourg EMT. Cardiovascular risk factors: differences between ethnic groups. Rev. Bras. Enferm. [Internet], 2020 [cited 2019 Jun 12]; 73 (4): e20180918. DOI: https://doi.org/10.1590/0034-7167-2018-0918.

6. Santos RC, Silva MS. Living conditions and therapeutic paths in a quilombola community in Goiás. Saúde Soc. [Internet], 2014 [cited 2020 Jul 26]; 23 (3):1049-63. DOI: https://doi.org/10.1590/S0104-12902014000300025.

7. Santos VC, Boery EN, Pereira R, Rosa DOS, Vilela ABA, Anjos KF, Boery RNSO. Socioeconomic and health conditions associated with quality of life of elderly quilombolas. Texto Contexto Enferm. [Internet], 2016 [cited 2020 Jul 26]; 25 (2): e1300015. DOI: https://doi.org/10.1590/0104-07072016001300015.

8. Ministério da Saúde (Br). Política Nacional de Saúde Integral da População Negra [Internet]. Brasília; 2017. [cited 2020 Jul 26]. Available from: https://bvsms.saude.gov.br/bvs/publicacoes/politica_nacional_saude_populacao_negra_3d.pdf.

9. Ministério da Saúde (Br). Secretaria de Atenção à saúde. Envelhecimento e saúde da pessoa idosa [Internet]. Brasília; 2006. [cited $2020 \mathrm{Jul}$ 28]. Available from http://bvs.saude.gov.br/bvs/publicacoes/evelhecimento_saude_pessoa_idosa.pdf.

10. Associação Brasileira para o Estudo da Obesidade e da Síndrome Metabólica. Diretrizes Brasileiras de Obesidade/ABESO Associação Brasileira para o Estudo da Obesidade e da Síndrome Metabólica [Internet]. São Paulo; 2016. [cited 2020 Jul 28 ]. Available from: https://abeso.org.br/wp-content/uploads/2019/12/Diretrizes-Download-Diretrizes-Brasileiras-de-Obesidade2016.pdf.

11. Malachias MVB, Souza WKSB, Plavnik FL, Rodrigues CIS, Brandão AA, Neves MFT, et al. 7a Diretriz Brasileira de Hipertensão Arterial. Arq. Bras. Cardiol. [Internet], 2016 [cited 2020 Jul 28]; 107(3Supl.3):1-83. Available from: http://publicacoes.cardiol.br/2014/diretrizes/2016/05_HIPERTENSAO_ARTERIAL.pdf. 
12. American Diabetes Association. Approaches to glycemic treatment. Diabetes Care [Internet], 2016 [cited 2020 Jul 28]; 39 (Suppl. 1): S52-S59. DOI: https://doi.org/10.2337/dc16-S010.

13. Oliveira FMRL, Costa KNFM, Pontes MLF, Batista PSS, Barbosa KTF, Fernandes MGM. Risk factors associated with hospitalization in the elderly assisted in primary health care. Rev. enferm. UERJ [Internet], 2018 [cited 2020 August 2]; 1:e15488. DOI: http://dx.doi.org/10.12957/reuerj.2018.15488.

14. Santos DMS, Prado BS, Oliveira CCC, Santos MAA. Prevalence of Systemic Arterial Hypertension in Quilombola Communities in the State of Sergipe, Brazil. Arq. Bras. Cardiol. [Internet], 2019 [cited 2020 Jul 26]; 113 (3): 383-90. Available from: http://publicacoes.cardiol.br/portal/abc/portugues/2019/v11303/pdf/11303012.pdf.

15. Instituto Brasileiro de Geografia e Estatística (IBGE). Pesquisa Nacional por Amostra de Domicílios: um panorama da saúde no Brasil - acesso e utilização dos serviços, condições de saúde e fatores de risco e proteção à saúde: 2008 [Internet]. Rio de Janeiro; 2010. [cited $2020 \mathrm{Jul}$ 25]. Available from: https://biblioteca.ibge.gov.br/visualizacao/livros/liv44356.pdf.

16. Pereira JC, Barreto SM, Passos VMA. The health profile of elderly Brazilians needs to improve: a population-based study. Arq. Bras. Cardiol. [Internet], 2008 [cited 2019 Jun 12]; 91(1):1-10. DOI: https://doi.org/10.1590/S0066-782X2008001300001.

17. Diniz MA, Tavares DMS. Risk factors for cardiovascular diseases in aged individuals in a city in the state of Minas Gerais. Texto Contexto Enferm. [Internet], 2013 [cited 2019 May 14]; 22(4): 453-67. DOI: http://dx.doi.org/10.1590/s010407072013000400003.

18. Barbosa MEM, Bertelli EVM, Aggio CM, Scolari GAS, Marcon SS, Carreira L. Factors associated with adult/elderly adherence to the treatment of arterial hypertension in primary care. Rev. enferm. UERJ [Internet], 2019 [cited 2019 May 16 ]; $27: e 45894$. DOI: http://dx.doi.org/10.12957/reuerj.2019.45894.

19. Bezerra VM, Andrade ACS, César CC, Caiaffa WT. Quilombo communities in Vitória da Conquista, Bahia State, Brazil: hypertension and associated factors. Cad. Saúde Pública [Internet], 2013 [cited 2019 May 25]; 29 (9):1889-902. DOI: https://doi.org/10.1590/0102-311X00164912.

20. Oliveira SKM, Caldeira AP. Risk factors for non-communicable chronic diseases in quilombo communities in northern Minas Gerais, Brazil. Cad. Saúde Colet. [Internet], 2016 [cited 2020 Jul 29]; 24 (4): 420-427. DOI: https://doi.org/10.1590/1414$462 \times 201600040093$.

21. Soares DA, Kochergin CN. Factors associated with Obesity in Elderly Maroons in Bahia, Brazil. Rev. APS [Internet], 2017 [cited 2020 Jul 29]; 20 (2): 174-84. DOI: https://doi.org/10.34019/1809-8363.2017.v20.15768.

22. Almeida ILS, Santos SR, Queiroz BM, Mussi RFF. Lifestyle, morbidity and multimorbity in adult. ABCS Health Sciences [Internet], 2020 [cited 2020 Jul 29]; 1325 (45): 1-7. DOI: https://doi.org/10.7322/abcshs.45.2020.1325.

23. Ministério da Saúde (Br). Secretaria de Atenção à Saúde. Departamento de Ações Programáticas Estratégicas. Manual de atenção à mulher no climatério/menopausa [Internet]. Brasília; 2008 [cited 2020 Jul 26]. Available from: http://bvsms.saude.gov.br/bvs/publicacoes/manual_atencao_mulher_climaterio.pdf.

24. Gus I, Ribeiro JA, Kato S, Bastos J. Variations in the Prevalence of Risk Factors for Coronary Artery Disease in Rio Grande do SulBrazil: A Comparative Analysis between 2002 and 2014. Arq. Bras. Cardiol. [Internet], 2015 [cited 2020 Jul 30]; 105(6):573-9. Available from: https://www.scielo.br/scielo.php?script=sci_arttext\&pid=S0066-782X2015002500573. 